The habitable space: from the Modern Movement up to the present, rupture and continuity

PALABRAS CLAVE • MOVIMIENTO MODERNO • ESPACIO HABITABLE - TRANSFORMACIONES · CONTEMPORANEIDAD

KEYWORDS • MODERN MOVEMENT · LIVING SPACE TRANSFORMATIONS · CONTEMPORANEITY
RESUMEN

El centro histórico de la ciudad de Camagüey declarado Patrimonio Cultural de la Humanidad en el 2008, es un exponente de valores arquitectónicos que responden a diferentes etapas, dentro de ellas destaca la arquitectura del Movimiento Moderno y en específico los edificios de apartamentos construidos en la década de 1950.

La arquitectura de este período hizo aportaciones relevantes en la adaptación a las condiciones

climáticas locales y a una nueva organización espacial. El desconocimiento de este patrimonio ha ocasionado transformaciones derivadas de su adaptación a condicionantes sociales y económicas actuales. Su flexibilidad para adaptarse a nuevos usos es posible, siendo necesario un adecuado equilibrio que debe alcanzarse a partir de repensar la habitual relación entre pasado, presente y futuro. Este artículo forma parte de investigaciones desarrolladas sobre el tema en Tesis de Maestría y avances de Tesis Doctoral contenidas ambas en proyectos de investigación empresariales.

\section{ABSTRACT}

The historic center of the city of Camagüey, declared a UNESCO World Heritage Site in 2008, is an example of the architectural values that respond to different stages. Among the highlights is the architecture of the Modern Movement and, more specifically, the apartment buildings built in the 1950s. Important architectural contributions were made in this period regarding the adaptation to local climatic conditions and to new spatial organization. The lack of recognition of this heritage has caused transformations derived from its adaptation to current social and economic conditions.

Its flexibility to adapt to new uses is possible, but it needs an adequate balance that must be achieved as a rethinking of the usul relationship between the past, the present and the future. This article is part of research developed in the framework of the thesis of a Master's degree and progress toward a Doctoral thesis, both in the area of managerial investigation projects.

\title{
El espacio habitable
}

\section{Del Movimiento Moderno a la actualidad, ruptura y continuidad}

\author{
MG. DIANELIS FALLS VALDIVIESO · Universidad de Camagüey Ignacio Agramonte Loynaz, Camagüey, Cuba \\ dianelis.falls@reduc.edu.cu \\ DRA. MABEL TERESA CHAOS YERAS · Universidad de Camagüey Ignacio Agramonte Loynaz, Camagüey, Cuba \\ mabel.yeras@reduc.edu.cu
}

Fecha de recepción: 16 de enero de 2018 - Fecha de aceptación: 02 de octubre de 2018

\section{INTRODUCCIÓN}

Enfrentar la problemática de la valoración del patrimonio cultural exige disponer de instrumentos que permitan conocer la realidad para definirla, aprehenderla e identificarla, así como distinguir sus características y analizar sus variaciones a lo largo del tiempo, para situarla mejor en el presente. El conocimiento y reconocimiento del patrimonio cultural en un centro histórico debe enmarcarse dentro de las políticas de conservación para lograr su preservación y salvaguardia. Según el Manual para Inventarios de Bienes Culturales Muebles del Ministerio de Cultura de Colombia (López, García y Serpa, 201 1), en el mismo instante en que se fija la atención sobre una expresión de la cultura, hay valoración. En la Declaración de San Antonio se asegura que la autenticidad de nuestros recursos culturales yace en la identificación, evaluación e interpretación de los valores verdaderos como fueron percibidos por nuestros antepasados y por nosotros ahora en una desarrollada y diversa comunidad (Gómez y Peregrina, 2009).

El patrimonio cultural, como transmisor de un mensaje subjetivo del pasado de las naciones, permanece en la vida presente como testimonio de sus renovadas tradiciones. El demérito o destrucción de sus obras, en aras de un incierto progreso, supone una pérdida para su valoración a escala mundial. Unido a ello, la dinámica presente que caracteriza la actuación de la sociedad contemporánea ha llevado a una cultura de la inmediatez, en la que se desconoce el valor del patrimonio frente a las más diversas necesidades sociales. Aparece entonces la tensión entre recuperación y renovación, mantención y transformación del valioso patrimonio de nuestras ciudades.

Ante esta realidad Gómez y Pérez (2011) señalan que la conservación del patrimonio parte del reconocimiento de los valores de uno o varios de sus componentes legados desde el pasado, la posesión de cierto sentido de pertenencia sobre el mismo y el sentimiento de responsabilidad para protegerlo. En este sentido, otros autores observan que la búsqueda de valores debe atender en primera instancia, a aquellos reconocidos en la localidad, aquellos que se expresan en situaciones concretas y que conducen al hombre a su bienestar y perfeccionamiento (Chateloin, 2008). Este reconocimiento se relaciona con el nivel de bienestar que el inmueble brinda al hombre y en esa medida, se desarrolla un proceso de identificación de las personas con ese espacio. De esta forma, se comprende que los valores son cualidades especiales adicionadas a las características físicas por parte de un grupo social, lo que demuestra la subjetividad del concepto y la dificultad para establecer criterios de validez universal (Cárdenas, 2012). Los valores, sin embargo, no están todo lo 
precisados que convendría (Alfonso, 2014) siendo necesaria una articulación entre la protección legal y las premisas de diseño.

Atendiendo a ello, el proceso de valoración de las obras en su perspectiva de bien patrimonial, implica la participación no solo de los especialistas, sino del resto de las comunidades afectadas y es parte del problema actual, la ausencia de una apreciación de los valores espaciales, determinados por aspectos formales y ambientales. Un ejemplo de ello son los edificios de apartamentos del movimiento moderno (Rodríguez, 1997), que a partir de nuevas formas de construir mostraron valores tanto en la forma de vida como de hacer arquitectura. En este sentido, el valor arquitectónico se relaciona con el estilo y calidad de diseño, las formas, los usos y los tipos de materiales, cuando presenta cualidades destacables o relevantes en lo referente a calidad espacial, volumetría, proporciones y recorridos o calidad funcional, la coherencia espacial, uso correcto de los locales y confort (Alfonso, 2014).

En el caso de los valores espaciales puede afirmarse que el cambio de esta arquitectura no solo se manifiesta por su imagen y volumetría; también en su concepción planimétrica aparece una nueva organización de los espacios interiores (Ramírez 2008). Generalmente, el estar, el comedor y la cocina se unen en un solo espacio de intercambio fluido y permeable en relación directa con el exterior. El patio con vegetación, como corazón verde de la vivienda, deja de ser el elemento esencial para compartir con la envolvente el intercambio climático con el exterior. El intercambio se incrementa mediante amplias terrazas, algunas en voladizo que realzan el valor estético de estas obras.

Le Corbusier realizó importantes análisis sobre la concentración de funciones afines o iguales entre sí, dormitorios unidos, la ubicación de cocina al lado del comedor, la simplificación y reducción de circulaciones, entre otros. En sus obras un elemento fundamental fue la adaptación flexible: planta libre, mobiliario abatible, armarios empotrados o los intercomunicadores entre los espacios de salón y cocina. Según Segre (1988), estos núcleos habitacionales constituyen los aportes más significativos de la arquitectura y el urbanismo racionalista. Como plantea Gropius:

El problema de la vivienda mínima (minimal wohnung) consiste en establecer el mínimo elemental de espacio, de aire, de luz y de calor que necesita el hombre para poder desarrollar plenamente sus funciones vitales, sin limitaciones debidas a la vivienda misma, es decir, un mínimo modus vivendi en lugar de un modus non moriendi" (Benévolo, 1976, p. 590).

Pero el espacio arquitectónico no puede comprenderse como resultado del dimensionamiento interior generado por las actividades a realizar que se limitan en un espacio físico. El espacio arquitectónico también tiene que aportar al "hombre" otros elementos, ofreciendo las condiciones climáticas adecuadas y sintetizando un ambiente que a su vez satisfaga sicológica y culturalmente (Fernández, 1979). Por lo que, a partir de lo planteado por estos autores, se podría decir que el valor espacial de la arquitectura, es aquel reconocido por el hombre a partir de la forma en que es manejado, desde su concepción y que permite utilizarlo, aprovecharlo o valerse de él, por estar dotado de atributos y cualidades positivas especiales, que posibilitan el desarrollo de funciones y un potencial desempeño, según los requerimientos y necesidades de los usuarios. Tiene como base las concepciones, conductas, costumbres, en correspondencia con la forma de vida, condicionando formas de uso y de comportamiento.

Si bien la relevancia de la arquitectura y de la ciudad en el panorama del patrimonio cultural nacional es un criterio plenamente aceptado, reconocidas ambas como el legado más importante de la cultura material cubana (Rodríguez, 201 1), en lo concerniente a la arquitectura moderna, actualmente se intenta poner en evidencia los valores de ese extenso patrimonio, en gran medida afectado ya por la falta de un mantenimiento adecuado y por la poca conciencia de su valor debido a su corta edad, comparada con el importante patrimonio colonial. Ese enorme fondo construido con los códigos modernos no solo tiene un valor histórico y artístico, sino también un valor de uso. Además, muestra soluciones que todavía pueden ser válidas para una reinterpretación que combine la tradición reciente con una expresión contemporánea. En este sentido la Carta de Salta de 1982, plantea que la conservación del patrimonio arquitectónico no tiene validez si sus valores no son asumidos por la comunidad que es la responsable de su custodia y depositaria del mismo (Cárdenas, 1998).

En la ciudad de Camagüey, el lugar donde se insertaron los edificios de apartamentos que contribuyeron al enriquecimiento formal, otorgando un aire de modernidad, fue el centro histórico (Gómez, 2009). El poco reconocimiento de sus valores por parte de especialistas, instituciones y la población en general ha traído como consecuencia, varias transformaciones al interior y exterior de los mismos. En ocasiones los malos resultados de estas modificaciones, han estado asociados a la carencia de recursos económicos de la población que los habita, provocando su deterioro (Chaos, Mancebo y Falls, 2016). Por otro lado, como resultado de satisfacer sus necesidades individuales los habitantes realizaron modificaciones sin conocimientos técnicos, trayendo consigo la destrucción del patrimonio construido y la agresión a la integridad física de estas edificaciones, con consecuencias en muchos casos irreversibles.

Los edificios de apartamentos del movimiento moderno son la imagen de conceptos de vanguardia de la época, que se introdujeron en el centro histórico y brindaron una interpretación particular de la modernidad, motivo por el cual es importante la preservación de sus características iniciales. Sin embargo, en la actualidad gran parte de este repertorio evidencia un deterioro físico significativo debido a la falta de mantenimiento continuado, los usos incompatibles ubicados en la planta baja 
o en los apartamentos, las modificaciones inadecuadas en el interior producto de las necesidades de las familias y las entidades estatales, y por último, las transformaciones que se evidencian en fachada. La investigación desarrollada' constituye un paso más para el logro del reconocimiento de los valores de los edificios de apartamentos del Movimiento Moderno en la ciudad de Camagüey.

\section{OBJETIVOS Y METODOLOGÍA}

El objetivo de la investigación es evaluar y proponer alternativas arquitectónicas y constructivas para las edificaciones del centro histórico de Camagüey, que permitan mantener los valores de habitabilidad como una condición patrimonial de la arquitectura moderna para por una parte salvaguardar los las características de este patrimonio y, por otra, tenerlas como referentes para el desarrollo de nueva arquitectura.

La metodología general es cuantitativa y cualitativa y se desarrolla en tres fases:

FASE I. Estudios previos. Los estudios previos de la investigación comprendieron fuentes documentales y bibliográficas, recogidas de los fondos del Archivo Histórico Provincial de la ciudad Camagüey (AHPC), consultados para verificación de la información de los inmuebles a trabajar y documentar las transformaciones presentes en la actualidad. Para el procesamiento de este volumen de información se empleó el método de análisis de contenido.

FASE II. Diagnóstico. En esta fase se desarrolló el diagnóstico detallado de los inmuebles en estudio, mediante la consulta de fuentes directas; como la visita al terreno, para identificar las edificaciones intervenidas por los propietarios y verificar la calidad del habitar asociada a ello. En este segundo momento se empleó la observación estructurada como parte del trabajo de campo que tuvo como soporte material el análisis de documentos gráficos como (planos, fotografías, levantamientos) y escritos, para la posterior evaluación en la Fase III de las intervenciones realizadas en relación con los criterios de valor determinados por el equipo o por el instrumento formal.

En esta fase además se definieron de manera aleatoria casos de estudio y se consultó mediante una entrevista dirigida a las familias que habitan los inmuebles y a especialistas en relación con las características del espacio disponible. Esta entrevista tuvo como fin, conocer el nivel de satisfacción de la población residente, así como el reconocimiento de determinadas cualidades en los inmuebles que habitan y en el caso de los especialistas lograr definir aquellos elementos que permiten identificar sus valores.

FASE III. Evaluación y propuesta. En esta fase se realizó el análisis del programa y de los requerimientos contemporáneos de los habitantes de los apartamentos, para la elaboración de una propuesta de intervención arquitectónica y constructiva en dos casos de estudio, un edificio cuyos lineamientos de diseño reflejan el ideario del movimiento moderno, que ha sufrido transformaciones, pero que aún conserva sus principales características y el otro correspondiente a una intervención de nuevo edificio, que se integra al área protegida, utilizando como referentes algunas características de la arquitectura del movimiento moderno.

\footnotetext{
Antecede a la Tesis Doctoral titulada "Valores espaciales de los edificios de apartamentos del Movimiento Moderno en el centro histórico de Camagüey" que desarrolla la autora Dianelis Falls Valdivieso, la Tesis en opción al título de Máster en Conservación de Centros Históricos y Rehabilitación del Patrimonio Edificado "Caracterización de los edificios de apartamentos del Movimiento Moderno del centro histórico de Camagüey" (2017), bajo la tutoría de la Dra. C. Mabel T. Chaos Yeras; a partir de la cual y de los avances del Doctorado se desarrolla el presente artículo.
}

\section{LOS EDIFICIOS DE APARTAMENTOS DEL MOVIMIENTO MODERNO EN EL CENTRO HISTÓRICO DE CAMAGÜEY}

Una parte importante del centro histórico de Camagüey fue inscrito en la lista del Patrimonio Cultural de la Humanidad desde el 2008 por su excepcionalidad universal (Gómez y Pérez, 2011) al contar con una trama urbana irregular que dio lugar al amplio sistema de plazas y plazuelas como núcleos, entre los que sobresalen las Iglesias. Otra característica a valorar, fue la integridad visual de su arquitectura y la unidad dentro de una amplia diversidad de estilos arquitectónicos. Justamente dentro de esta variedad se encuentran los edificios de apartamentos del movimiento moderno. Además, se incluyen dentro de los elementos a destacar las características de sus calles estrechas de trazado curvilíneo que dan lugar a espacios de múltiples formas que logran una integridad urbana y unido a ello el empleo del barro como material local en la elaboración de ladrillos, tejas, pisos, tinajones y obras de arte. Este conjunto patrimonial cuenta con un considerable repertorio de edificios de apartamentos construidos en los años 1950, que recogen la tradición constructiva que refleja la identidad cubana, en la búsqueda de una arquitectura que se adaptara a las condiciones locales, mediante soluciones inspiradas en el movimiento moderno internacional que irrumpió en la trama urbana tradicional del centro histórico camagüeyano. De vital importancia resulta el análisis de los edificios de apartamentos del movimiento moderno como referente de una arquitectura que pensó en dar bienestar al hombre elevando su calidad de vida con soluciones arquitectónicas novedosas, sin embargo aún no se reconocen del todo, las características de su impronta que determinaron en otro tiempo, la contemporaneidad de la ciudad.

Estos inmuebles geometrizaron la imagen de la ciudad tradicional y si bien, no se generaron grandes torres de apartamentos, sí se modificó el perfil predominante de edificaciones de uno o dos pisos para llegar a tener tres y en algunos 
casos cinco, siete y once pisos. Aunque, cabe señalar, se intentó ser respetuoso con ese perfil, pues el mayor porcentaje de estos edificios se mantuvo con un predominio a la horizontalidad, lo cual puede considerarse un seguimiento del pensamiento de Le Corbusier para algunas de sus intervenciones, cuando plantea que la estrategia del proyecto nace del respeto por la ciudad, de no intentar destacar ni romper su perfil urbano, según lo expuesto por Delgado (2017). De igual forma quedaron modificadas las proporciones de vanos y puntales. De forma general estas edificaciones se insertaron dentro de la trama urbana del centro histórico, en algunas soluciones aparece en el primer piso, recintos para rentar o negocios, estos espacios buscaban la transparencia mediante el empleo de amplias superficies de cristal localizadas desde el piso al cielo.

El centro histórico de Camagüey se divide en ocho zonas de protección (FIGURA 1), de las cuales la zona dos presenta un mayor número de edificios de apartamentos que se pueden identificar con movimiento moderno.

En el desarrollo del estudio se selecciona como primer caso, el edificio de apartamentos ubicado en la calle General Gómez, № 3 entre República y Avellaneda construido en el año 1955-1957, donde es posible comprobar a partir de las fuentes documentales de archivo ${ }^{2}$ el grado de transformación sufrido por intervenciones realizadas por el grupo residente, pero que aún conserva características espaciales de los apartamentos originales. Su expresión formal esta lograda mediante la intersección de planos y el juego de llenos y vacíos en la fachada. En el interior es posible verificar la flexibilidad de los espacios, la agrupación de funciones afines, la jerarquización de áreas en la estructura general de la vivienda, permitiendo de esta forma, mayor iluminación y ventilación en los lugares que lo requieren, siendo estas
1. Centro histórico de Camagüey con la división de las ocho zonas de protección. Imágenes con algunos exponentes del Movimiento Moderno de la zona de protección 2. Elaboración de las autoras.

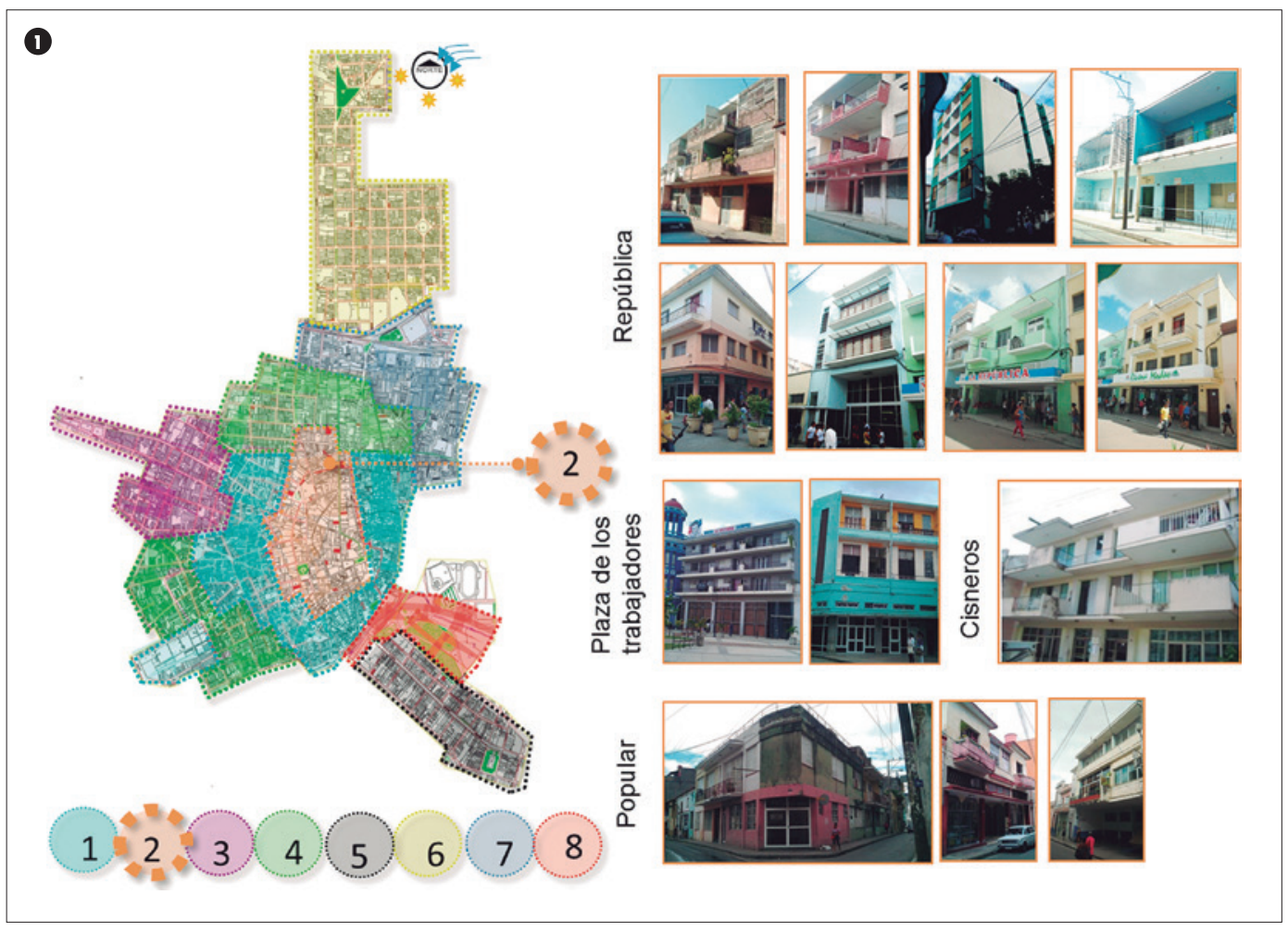

las características valoradas por la población que lo habita, de acuerdo a la información extraída a partir de las encuestas realizadas. Este reconocimiento del valor de varios aspectos arquitectónicos, constituyen referentes para recuperar el estado de bienestar logrado en esos inmuebles del pasado, en las nuevas inserciones de espacios habitables, en el área de la ciudad valorada patrimonialmente.

No cabe duda de que la organización del espacio habitable en el contexto de la cultura contemporánea, provoca divergencias y convergencias entre las soluciones deseadas por la comunidad y las consiguientes respuestas entregadas por la arquitectura, sin embargo, las soluciones son posibles desde un análisis que contemple el reconocimiento de los valores de la arquitectura del pasado, la impronta de las necesidades de la sociedad contemporánea y una visión de futuro en vista a la velocidad de cambio a la que debe dar siempre respuesta la arquitectura.

Por estas razones, se incluye en el análisis un segundo caso, donde se interviene una edificación localizada dentro del área protegida en el centro historio de la ciudad Camagüey, creando un nuevo edificio de apartamentos con tecnologías contemporáneas a partir de una lectura de los referentes formales y espaciales del movimiento moderno. Este edificio se inserta en uno de los ejes viales de mayor importancia de la ciudad por su cercanía al centro fundacional y en un lote muy transformado por las sucesivas subdivisiones de la unidad edificatoria original. El nuevo edificio se propone en Independencia No. 105, 107, 109, 109A, 109B y 109 Altos. 


\section{PATRIMONIO TRANSFORMADO}

El primer caso de estudio ya mencionado, correspondiente al edificio de apartamentos ubicado en la calle General Gómez, № 3 y construido en el año 1955-1957, debido a las transformaciones efectuadas por sus habitantes ha perdido en el primer piso las condiciones de habitabilidad presentes en los pisos superiores no intervenidos. Este primer piso, según los planos originales del AHPC se pudo comprobar que no estaba destinado al uso residencial sino a actividades anexas, por lo que actualmente no cumple con los requerimientos necesarios para su nueva función. Por este motivo, la propuesta asume la reorganización de los espacios para lograr una mejor calidad de vida de sus habitantes, formando parte de un proyecto académico-profesional, que identifica e interpreta el valor de los edificios y los requerimientos de los usuarios, en vista a propiciar intervenciones de mayor coherencia con los valores de la arquitectura moderna que la comunidad reconoce como un aporte a la habitabilidad y a la calidad de vida. Estos son: iluminación, flexibilidad espacial, ventilación.

El inmueble en estudio consta de tres pisos (FIGURA 2), el primero originalmente se reservó para áreas de renta, contando con tres espacios disponibles, el segundo y tercer piso tienen cinco apartamentos cada uno (Falls, D., 2016). El edificio presenta su fachada principal a la calle General Gómez con una orientación noreste. Los trabajos de construcción del mismo fueron comenzados en el año 1956 y concluidos en 1957, con un área de $972.10 \mathrm{~m}^{2}$ construidos. El proyectista principal fue el arquitecto Ricardo Rodríguez, también intervino el arquitecto Kemel Suarez y el propietario original fue el señor Jorge Recio Rodríguez.

Su diseño es sencillo, con uso de trazos simples y líneas rectas, así como de volúmenes prismáticos asumiendo códigos formales propios del movimiento moderno. Para su construcción se empleó el ladrillo como material a vista lo que proporciona una decoración sencilla basada en el uso de materiales locales, lo cual evidencia

2. Caso de estudio 1. General Gómez, N 3 entre República y Avellaneda. Plano de fachada original, AHPC, Fondo Ayuntamiento, Legajo: 244, Expediente $N^{\circ}$ 3, 4, 5 (izquierda). Fotografía de las autoras 2018 (derecha).

3. Utilización de material a vista y algunas de las afectaciones presentes en el inmueble (de izquierda a derecha). Fotografía de las autoras.

4. Solución espacial original del primer nivel del inmueble (izquierda) y actual (derecha). Fuente: Planta elaborada a partir de planos originales del AHPC. Anisleimis Brito Álvarez y José Carlos Poleo Zaldívar en colaboración con las autoras.
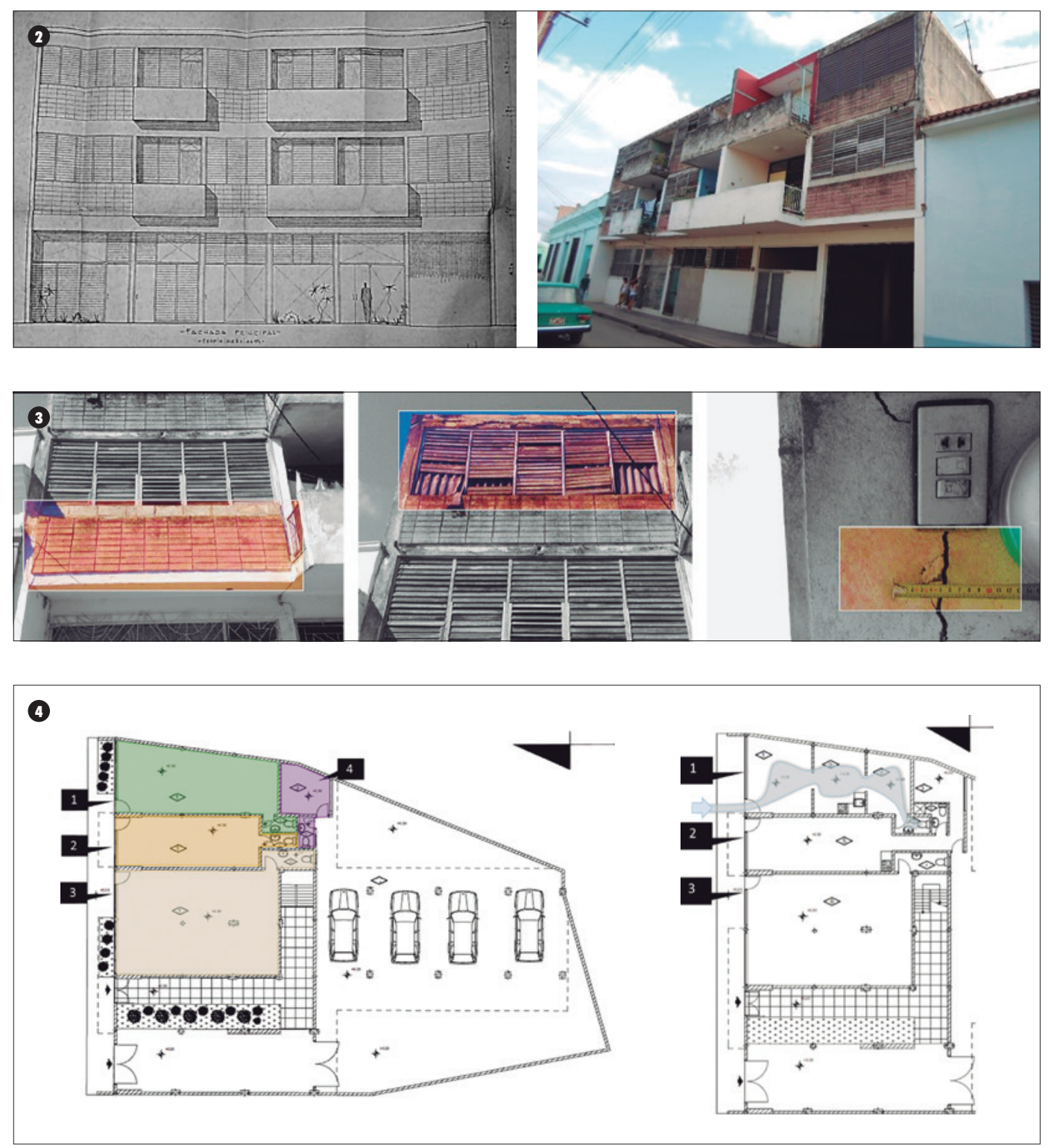

la adaptación formal al contexto inmediato. El inmueble se encuentra en un mal estado constructivo, por la falta de mantenimiento y porque las remodelaciones han sido realizadas por los residentes sin la adecuada asesoría técnica. El estado actual de la edificación afecta las vistas exteriores y las transformaciones llevadas a cabo hacia el interior han modificado en algunos casos los vanos y la carpintería original, provocado afectaciones en algunos muros y también se aprecian filtraciones en la losa de entrepiso (FIgURA 3).

Los tres espacios en planta baja, concebidos originalmente para renta, contaban con un pequeño servicio sanitario cada uno, ubicados de forma concentrada para economía de las instalaciones y un cuarto destinado para 
5. Transformaciones exteriores del edificio. Fotografías de las autoras.

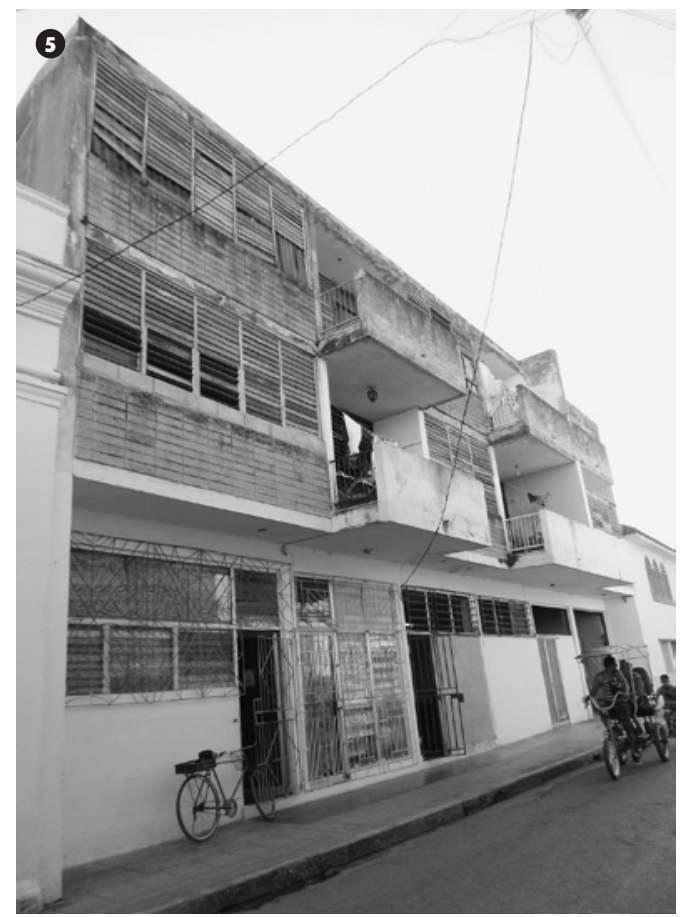

el encargado del edificio con entrada por el área de parqueo. Estos espacios han sufrido modificaciones, destinándose dos de ellos para uso habitacional y otro para uso administrativo. En la actualidad, los espacios 1 y 2 están ocupados por familias, en el caso del apartamento 2 se incorporó el área originalmente destinada al encargado del edificio y el espacio 3 es utilizado por la Oficina del Sindicato de Comercio (Figura 4).

En el análisis realizado al apartamento 1 se observó que presenta exteriormente la incorporación de rejas en puerta principal de acceso y ventanas como protección. Dichos diseños nada tienen que ver con la posible reinterpretación de las características de la arquitectura moderna aquí relevadas. Al interior se aprecia la falta de ventilación e iluminación natural por la ausencia de vanos o ventanas, al no haber sido este local concebido originalmente para la función de apartamento y por la mala adaptación del espacio original a la nueva función, lo que atenta contra el bienestar

6. Vivienda adicionada en la azotea. Fotografía de las autoras.

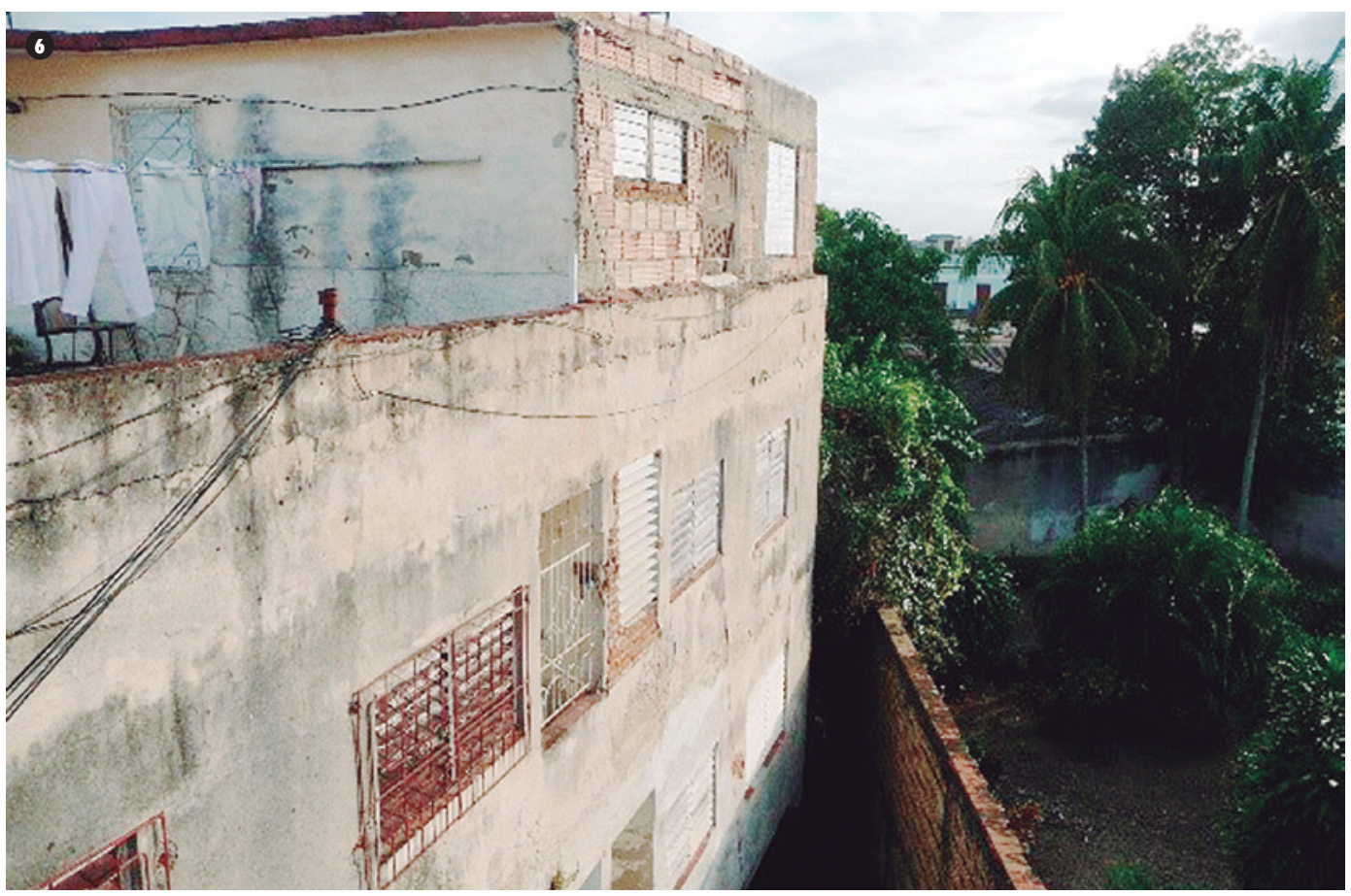

de los habitantes. De igual forma los residentes transformaron el espacio original, creando divisiones interiores para dar respuesta a los espacios imprescindibles como el de la cocina (FIgURA 5).

Derivado de la misma necesidad de espacios para uso residencial se incorporó un apartamento en la azotea del edificio que ha causado daños estructurales a los apartamentos que se ubican en el nivel inferior (FIGURA 6). De igual forma se evidencia que las modificaciones realizadas por los residentes no han contado con la asesoría necesaria y se han dado soluciones incompatibles que terminan poniendo en peligro la integridad física del bien patrimonial.

Si bien es cierto que las edificaciones deben, como principio de diseño, poder adaptarse, a partir de una adecuada flexibilidad a los requerimientos de la vida moderna; ello no debe atentar contra su preservación. Las soluciones deben ser pensadas siempre que se consideren los valores propios del bien y se realicen propuestas logrando un equilibrio entre pasado, presente y futuro considerando la preservación y adaptación a necesidades contemporáneas.

Para dar solución a las afectaciones causadas por el proceso de intervención de los espacios a través del tiempo y como muestra de que existen alternativas viables en la preservación de su originalidad y características afines, en las soluciones que aquí se presentan se plantea el respeto hacia el pasado del inmueble y a la vez la adaptación de los espacios a las exigencias actuales. Para ello se propone la reorganización funcional y espacial de las zonas modificadas. Se atienden principalmente las viviendas añadidas en el primer nivel en los antiguos locales de renta para negocio, a fin de dotarlas de adecuadas condiciones ambientales y espaciales para su nuevo uso como viviendas, garantizando un mejor confort.

De igual forma se propone la demolición de la vivienda construida en la azotea por el daño 
7. Propuesta para la reorganización funcional de los espacios en planta baja (Izquierda) y propuesta de fachada (Derecha). Anisleimis Brito Álvarez y José Carlos Poleo Zaldívar en colaboración con las autoras.

estructural que causa al edificio, eliminando el uso actual de Oficina del Sindicato de Comercio en el local de la planta baja para convertirlo en vivienda para los habitantes del apartamento incorporado en la azotea. Este nuevo espacio tuvo en cuenta las características de ese núcleo familiar. La función de oficina no requiere su ubicación en una zona privilegiada del centro de la ciudad y según el Plan Maestro de la Oficina del Historiador de la ciudad de Camagüey (OHCC) debe priorizarse la función del habitar y eliminar funciones administrativas.

En el caso del apartamento 1 se consideró necesario agregarle un espacio que originalmente era el cuarto del encargado del edificio y calar la losa de cubierta para generar un patinejo de ventilación al interior y fundamentalmente para el área de servicio sanitario y cocina. En el apartamento 2 se planteó la reubicación del servicio sanitario y la unidad de las instalaciones, agrupadas con las de la nueva vivienda propuesta. También se propuso como solución para la ventilación incorporar un patinejo. Por último, en el apartamento 3, que era el espacio que ocupaba la Oficina del Sindicato de Comercio, se propuso una solución con dos dormitorios, servicio sanitario, área común de cocinacomedor, este último con comunicación directa al exterior por el pasillo lateral de acceso al edificio. También se trabajaron las áreas libres del edificio, con vistas a crear un patio de usos múltiples y un espacio para lavaderos comunes a las tres viviendas de la planta baja. Adicionalmente está prevista la sustitución de todas las redes técnicas y la carpintería por su estado de deterioro (proyecto ya elaborado por la Empresa Provincial de Arquitectos de la Comunidad en Camagüey). Además se propuso el saneamiento de muros y la pintura con un estudio de color adecuado para este tipo de edificio moderno en consonancia con las regulaciones establecidas por la OHCC para el centro histórico. A partir de estos criterios se elaboraron variantes teniendo como base el estudio del contexto y la revisión del repertorio (Figura 7).

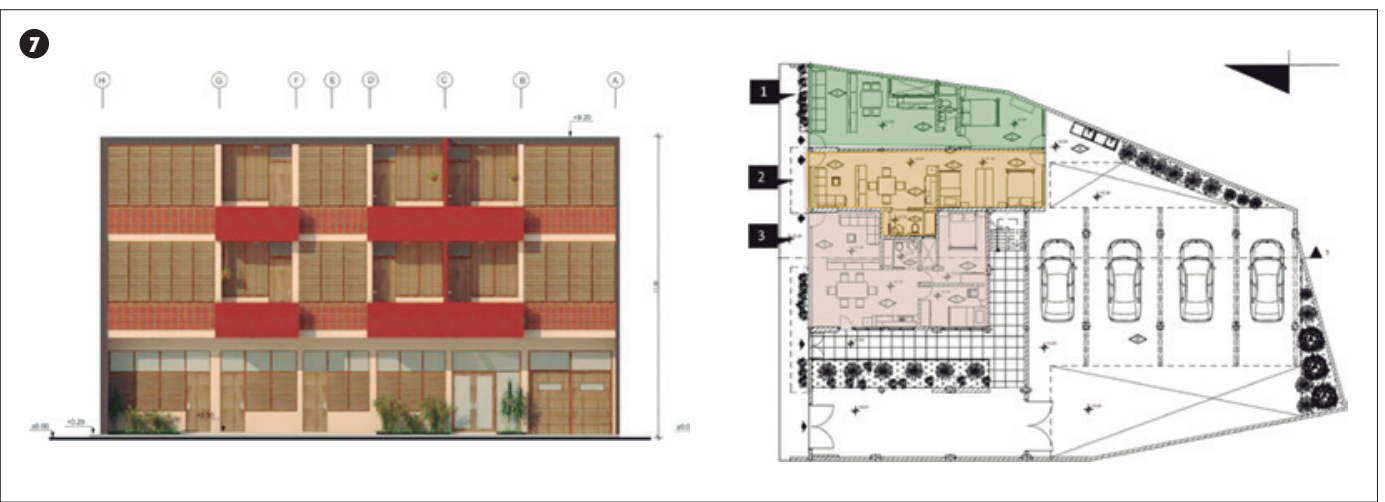

La propuesta permite mejorar las condiciones de vida de los habitantes y la imagen actual del edificio, pudiendo convertirse en referente para la intervención de otros valiosos exponentes del patrimonio moderno que de igual forma hayan sufrido transformaciones. El conocimiento de los valores de esta arquitectura permite además desarrollar nuevas propuestas para el hábitat en contextos de valor.

\section{EL MOVIMIENTO MODERNO, IMPORTANTE REFERENTE PARA NUEVAS INSERCIONES}

El segundo caso de estudio corresponde a la intervención en un área de valor patrimonial, del centro historio de la ciudad Camagüey creando un nuevo edificio de apartamentos con tecnologías contemporáneas a partir de una lectura de los referentes formales y espaciales del Movimiento Moderno. Si se considera que las características concretas de una vivienda dependen del clima, el terreno, el espacio, los materiales disponibles, de las técnicas constructivas que se utilicen, entre otros factores, así como los recursos económicos de sus propietarios, la combinación coherente de estos elementos puede dar lugar a condiciones óptimas de habitabilidad.

Dada la necesidad que todas las personas tienen de un alojamiento adecuado, este ha sido desde siempre un tema prioritario no solo para los individuos, sino también para los gobiernos. En los centros históricos las unidades edificatorias heredadas de otras épocas a menudo resultan poco flexibles desde el punto de vista espacial y, aunque es posible aumentar la capacidad de espacios, muchas veces no se obtienen intervenciones que satisfacen las condiciones ambientales y funcionales, y en la mayoría de los casos se afecta irreparablemente la imagen urbana de la ciudad por la pérdida de valores de ese bien patrimonial transformado.

La habitabilidad que las edificaciones modernas y sus características formales, espaciales y ambientales brindan, puede alcanzar vigencia, en este caso mediante sistemas constructivos prefabricados, propiciando los valores que las personas reconocen como favorables. Así se explica la necesidad del uso de nuevas tecnologías para edificios de viviendas en centros históricos, con sistemas prefabricados que permitan reducir los tiempos de ejecución por la complejidad funcional de sus emplazamientos, sin que ello vaya en detrimento de los valores del contexto.

Asumir el patrimonio desde un enfoque contemporáneo, buscando la mayor calidad de vida de sus habitantes y con ella, la integración social necesaria para el desarrollo sostenible de la ciudad; incluye también la incorporación de nueva arquitectura, que sea respetuosa y establezca un diálogo equilibrado con el mismo de manera que resuelva las 
necesidades contemporáneas y preserve los valores del contexto. En este sentido los aportes del movimiento moderno en cuanto a su flexibilidad y otros valores espaciales debe ser también un referente para la nueva arquitectura habitacional.

Se ha seleccionado, a manera de ejemplo, la unidad edificatoria ubicada en el eje Independencia con los números 105, 107, 109 , 109A, 109B y 109 Altos, del centro histórico de Camagüey, la cual ha sido transformada al pasar de los años espacial y volumétricamente. Este eje delimita físicamente uno de los laterales de la plaza mayor, actual Parque Agramonte, considerado como el centro fundacional del siglo XVI, con una función habitacional predominante en el $82 \%$ que exhibe importantes valores histórico-sociales y arquitectónicos dado por la diversidad de las tipologías presentes en el mismo, pese presentar varias y notorias transformaciones. El caso de estudio posee notorias modificaciones en su fachada, que han ocasionado la pérdida de sus valores originales (Figura 8).

De igual forma se han producido transformaciones en el espacio interior del inmueble a partir de las sucesivas divisiones del lote original por necesidades de sus habitantes y la falta de asesoramiento para lograr soluciones más adecuadas. Se presenta como alternativa de solución un programa arquitectónico que responde a la función de edificio de apartamentos de dos niveles. Este fue realizado teniendo en cuenta las especificidades del sistema PREFLEX. Uno de los últimos sistemas constructivos desarrollados en el país es el sistema conocido Prefabricado Flexible, su nombre deviene del criterio de flexibilidad en producción y diseño, ya que en las plantas de prefabricado los moldes podrán ser utilizados para producir varios componentes de un mismo tipo, pero con diferentes dimensiones, beneficiando la reducción de la cantidad de moldes en el 51,5\%; a la vez que pueden diseñarse pequeños módulos combinables entre ellos, dando así una gran gama de posibilidades de diseño.
8. Transformaciones de la vivienda 105, 107, 109, 109B, 109A, 109 Altos. Fotografía de las autoras.

9. Estado actual del interior del lote (Izquierda) y propuesta de solución (Derecha). Francis Torres González, bajo la asesoría de M. Teresa Chaos Yeras.
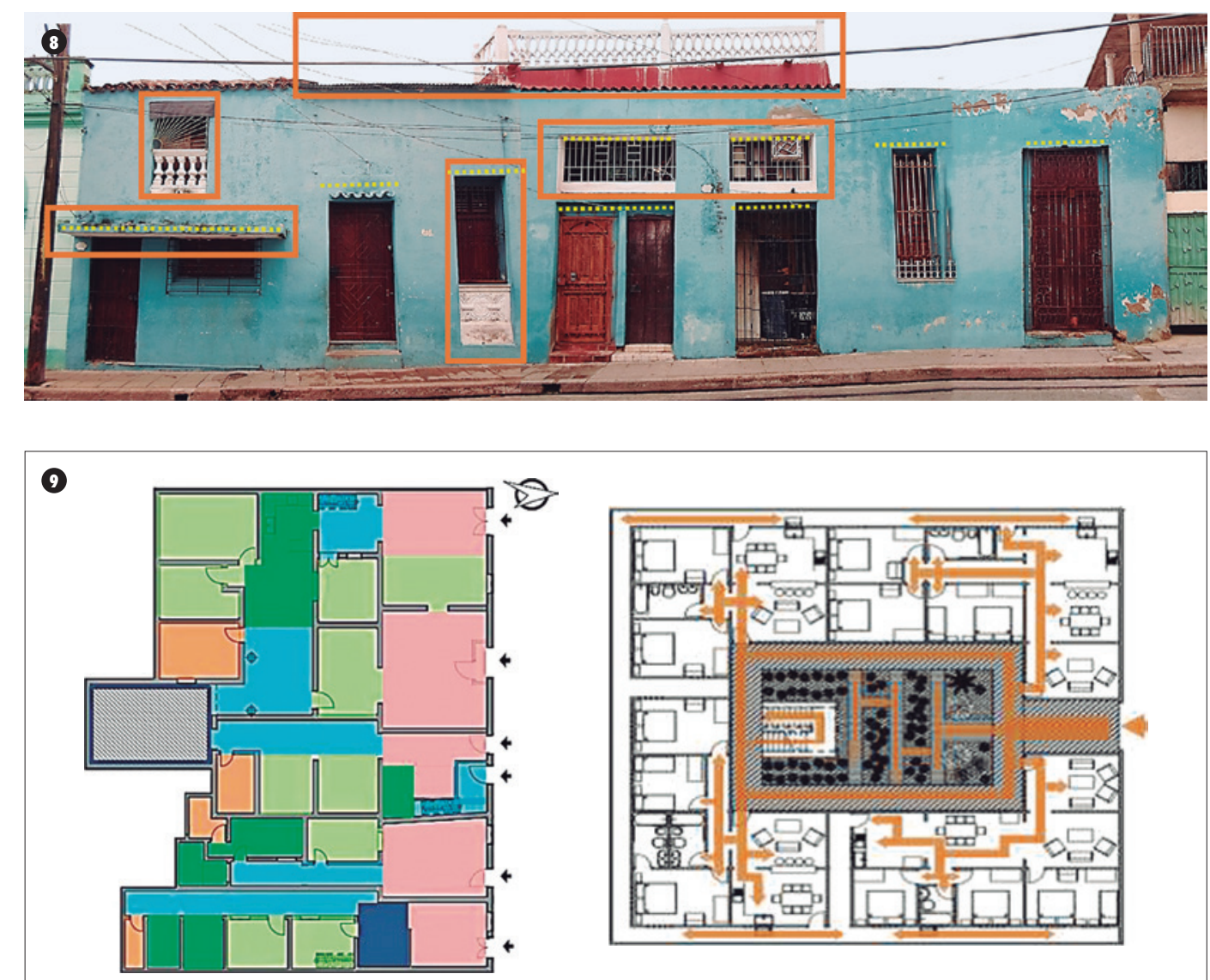

La planta del edificio se adapta a la forma del lote para un aprovechamiento máximo del espacio. La solución propone una entrada principal al edificio con un pasillo central que da acceso a la galería y a través de esta a los apartamentos, lo que permite una circulación cómoda a través de la edificación. Se proyectaron apartamentos independientes para aprovechar al máximo el espacio y el puntal propuesto de 4 metros en el primer nivel y 3 en el segundo, ello atiende a los puntales de las edificaciones colindantes. Se añadieron galerías y patinejos lo que brinda mayor organización espacial en la edificación y mejores condiciones de ventilación e iluminación natural.

Se hizo posible, debido a la amplitud, el aumento del número de viviendas existentes, teniendo como resultado 8 apartamentos con condiciones óptimas de habitabilidad, siendo ocupado el inmueble por un total de veintiocho personas. Las viviendas propuestas poseen áreas que oscilan entre los $90 \mathrm{~m}^{2}$ y $110 \mathrm{~m}^{2}$, contando con los espacios necesarios para su debido funcionamiento: sala, comedor, cocina, baño, dormitorio y patio de servicio. El número de espacios fue determinado según el tipo de núcleo familiar, y fueron utilizadas dimensiones medias para garantizar el uso de un mobiliario estándar. Se respeta la composición de las cinco familias que actualmente residen en el lote, destinandose cinco de los apartamentos para ellas (FIGURA 9).

Se muestra en la propuesta cómo han sido tomados como referentes los valores espaciales 
10. Propuesta de solución en fachada. Antes (izquierda), después (derecha). Francis Torres González, bajo la asesoría de M. Teresa Chaos Yeras.

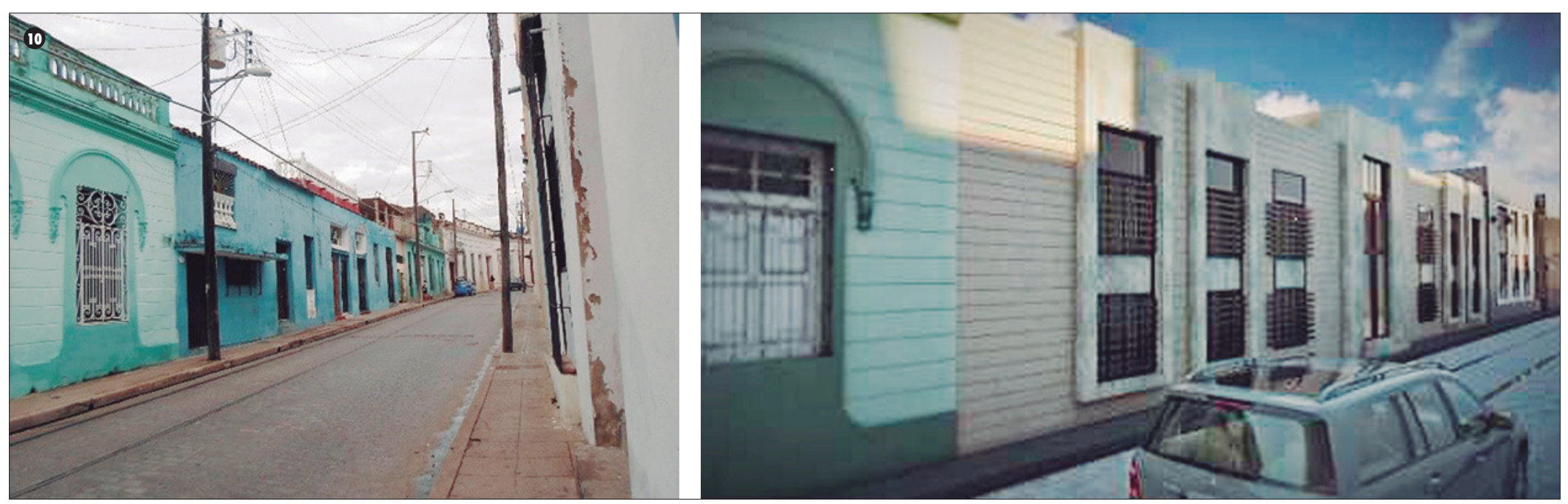

de la arquitectura identificada en el movimiento moderno, mediante el diseño de apartamentos con soluciones variadas para diversos tipos de familias, pero en todos los casos se aprovecha el espacio al máximo. Se logran agrupar las áreas afines como es el caso de la cocinacomedor, también se relacionan los dormitorios con el baño y a la vez se retiran a un área más privada dentro del área general. De forma general es posible alcanzar soluciones en algunos apartamentos donde en una sola pared húmeda quedan ubicadas las instalaciones de la cocina y el baño. La presencia del patio interno contribuye a la iluminación y ventilación de todas las unidades. Por otro lado, se llegan a configurar soluciones con un número mayor de espacios que los de las viviendas actuales y alcanzar un mayor confort. En algunos apartamentos se logran independizar los dormitorios de las áreas comunes y se incorpora un acceso exterior desde la cocina. Lo anterior permite evidenciar la continuidad de patrones validos de arquitecturas del pasado en soluciones del presente. Finalmente, la expresión formal del edificio realiza una transición hacia los criterios contemporáneos mediante la utilización de la línea recta simplificando y modernizando elementos figurativos en base a la lectura que se hace del contexto (FIGURA 10).

\section{CONCLUSIONES}

La arquitectura del movimiento moderno sin dudas representó un adelanto en el arte de construir y el habitar, aportando soluciones encaminadas a dar bienestar a los residentes a partir de la nueva organización espacial, la incorporación de elementos de control ambiental, las nuevas tecnologías y materiales de construcción y las soluciones formales. Sin embargo, el paso del tiempo, la falta de mantenimiento y las necesidades de la población propias de la dinámica de la vida actual, han llevado al deterioro y transformación de tan importante patrimonio.

De forma general en el primer caso de estudio se evidencian las consecuencias nefastas de la incidencia de los factores antes mencionados que van en contra de la preservación del patrimonio, el grado de transformación presente tanto en la fachada principal del inmueble como al interior del mismo. En el segundo caso ante las transformaciones irreversibles efectuadas a los inmuebles en contextos de valor, se plantea la posibilidad de crear una nueva arquitectura capaz de lograr mejor calidad de vida para las personas, al tener en cuenta elementos como las dimensiones, las relaciones espaciales y las condiciones ambientales de los locales entre otras.

Las dos propuestas ejemplifican las alternativas viables de solución tanto para el deterioro del valioso patrimonio del movimiento moderno, así como las posibilidades de la arquitectura de este período de servir como referente para nuevas intervenciones con la utilización de sistemas constructivos actuales que posibilitan diseños flexibles y adaptables a las necesidades contemporáneas. En ambos casos se da prioridad a las condiciones óptimas de habitabilidad al poner al hombre en el centro de las propuestas mediante la respuesta a sus necesidades actuales.

\section{REFERENCIAS BIBLIOGRÁFICAS}

Alfonso, A. (2014). Conservación del Patrimonio Cultural Inmueble. La Habana. Félx Varela.

Alfonso, A. (2014) Legislación y patrimonio inmueble. Antecedentes y aplicación en La Habana.

Arquitectura y Urbanismo, № 2, mayo, pp

5-18, Instituto Superior Politécnico "José Antonio Echeverría".

Benévolo, L. (1976). Historia de la arquitectura moderna. La Habana. Edición Revolucionaria. Instituto Cubano del Libro. 
Cárdenas, E. (1998). Problemas de teoría de la arquitectura. Guanajuato: Facultad de Arquitectura. Universidad de Guanajuato.

Cárdenas, E. (2012). Legado y diversidad en arquitectura y urbanismo. Arquitectura y Urbanismo, 28(1), 69-73.

Chateloin, F. (2008). El patrimonio cultural urbano y el criterio de centro histórico caso de estudio ciudad de la Habana. Tesis de Doctorado, Instituto Superior Politécnico José Antonio Echeverría. La Habana.

Chaos, M. T., Mancebo, I. y Falls, D. (enero-junio 2016). Participación comunitaria en el desarrollo del turismo urbano sostenible y responsable en ciudades Patrimonio de la Humanidad. Revista Monteverdia, 9 (1), 6. 48-59, ISSN:2077-2890

López M., García, C., Serpa, E. (2011). Manual para Inventarios de Bienes Culturales Muebles del Ministerio de Cultura de Colombia. Bogotá. Imprenta Nacional.

Delgado, E. (2017). Ciudad y agua. El contexto geometrizado del Hospital de Venecia de Le
Corbusier. Revista de Arquitectura. 22, (32), 32 41 ISSN 0716-8772 ISSN: 0719-5427

Falls, D. (2016). Los edificios de apartamentos del Movimiento Moderno en Camagüey: un valioso patrimonio a conservar desde los proyectos y con la Participación social. Revista Arquitecturas del Sur. 34, (49), 61. ISSN Impresa 0716-2677/ ISSN Digital 0719-6466

Fernández, J. M. (1979). La vivienda en Cuba. Historia - ensayo. Instituto Cubano del libro. Editorial Arte y Literatura, La Habana.

Gómez, L., Pérez, K. (2011). Reflexiones sobre patrimonio cultural. Lo inmaterial del centro histórico de Camagüey, patrimonio mundial. Apuntes. Revista De Estudios Sobre Patrimonio Cultural, 24(2).

Gómez, L., Peregrina, A. (2009). Documentos internacionales de conservación y restauración, Declaración de San Antonio, INAH y CONACULTA, México.

Gómez, L. (2009). El Centro Histórico Urbano de Camagüey, Patrimonio Mundial. Planeamiento, gestión y manejo. Apuntes. Revista De Estudios Sobre Patrimonio Cultural, 22(2).

Ramírez Li, R. (2008). Mirada al Movimiento Moderno cubano: un enfoque climático. Reparto Alturas del Vedado, Ciudad de La Habana. Apuntes. Revista De Estudios Sobre Patrimonio Cultural, 21 (2). Recuperado a partir de http://revistas. javeriana.edu.co/index.php/revApuntesArq/ article/view/8956

Rodríguez, E. (201 1). La Arquitectura del Movimiento Moderno. Selección de Obras del Registro Nacional. La Habana. Ediciones UNION.

Rodríguez, E. L. (1997). La década incógnita. Los cincuenta: modernidad, identidad, y algo más. Arquitectura Cuba (376) p. 43.

Segre, R. (1988). Lectura crítica del entorno cubano. La Habana. Letras Cubanas. 